\title{
EVALUACIÓN DE MÉTODOS DE FOURIER Y MÁXIMA ENTROPÍA PARA LA DETECCIÓN AUTOMÁTICA DE LA ENFERMEDAD DE PARKINSON
}

\author{
ASSESSMENT OF FOURIER METHODS AND MAXIMUN ENTROPY \\ FOR AUTOMATIC DETECTION OF PARKINSON 'S DISEASE
}

Juan Manuel Arias Mejía ${ }^{1}$, Elkyn Alexander Belalcázar Bolaños² ${ }^{2}$ Juan Rafael Orozco Arroyave ${ }^{3}$, Julián David Arias Londoño ${ }^{4}$, Jesús Francisco Vargas Bonilla ${ }^{5}$.

\footnotetext{
${ }^{1}$ Estudiante de Ingeniería Electrónica, integrante del Grupo de Investigación en Telecomunicaciones Aplicadas (GITA) Universidad de Antioquia.

2 Estudiante de Maestría en Ingeniería de Telecomunicaciones, integrante del Grupo de Investigación en Telecomunica ciones Aplicadas (GITA), Universidad de Antioquia

${ }^{3}$ Profesor asistente del Grupo de Investigación en Telecomunicaciones Aplicadas (GITA), Universidad de Antioquia.

4. Profesor asistente en el grupo de investigación en Simulación de Comportamiento de Sistemas (SICOSIS), Universi dad de Antioquia.

5. Profesor Asistente del grupo de investigación en Sistemas Embebidos e Inteligencia Computacional (SISTEMIC), Universidad de Antioquia.
}

Recibido: 07 de Octubre de 2014 Aceptado: 18 de Noviembre de 2014

*Correspondencia del autor: Juan Manuel Arias Mejía, Calle 70 No. 52-21, Medellín Colombia. E-mail: manuel.arias@udea.edu.co

\begin{abstract}
RESUMEN
La Enfermedad de Parkinson (EP) es la segunda condición clínica neurodegenerativa más prevalente después del Alzheimer y para el sistema de salud mundial es fundamental identificar marcadores tempranos; sin embargo en la actualidad es un campo nuevo de estudio que necesita un mayor desarrollo. Se ha demostrado que cerca del $90 \%$ de los pacientes con EP también desarrollan deficiencias en la voz, mostrando síntomas como un habla monótona, baja intensidad en el tono, pausas aisladas, pronunciación imprecisa de consonantes y problemas en la prosodia; y aunque dichos problemas están identificados solo del 3\% al 4\% reciben un tratamiento para la voz.

En el ámbito de la investigación, el análisis tiempo-frecuencia ha demostrado ser una herramienta poderosa en el procesamiento de señales acústicas, más específicamente, el procesamiento de la voz. Con el ánimo de tener representaciones limpias del espectro que ayuden a mejorar la extracción de características y mitigar otros problemas resultantes al usar métodos clásicos de estimación, se busca estudiar el comportamiento del Método de Máxima Entropía (MEM) comúnmente usado en ciencias oceanográficas y astronomía, en el estudio en señales de voz. Para estos experimentos fue usada una base de datos de pacientes que presentan la enfermedad de Parkinson usando 50 registros de voces patológicas y la misma cantidad de voces sanas, donde se analizaran segmentos sonoros y no sonoros obtenidos de la palabra "PA-TA-KA", sobre los cuales fueron estimadas energías provenientes tanto de la transformada de Fourier, como del método de máxima entropía. Con el fin de evaluar el rendimiento de los métodos clásicos y MEM se usó un clasificador K-nn y se encontraron tasas de acierto cercanas al $60 \%$ al considerar MEM tanto en fonemas como en sílabas.
\end{abstract}

Palabras Clave: Energía, enfermedad de Parkinson, espectro, K-nn, máxima entropía. 


\begin{abstract}
Parkinson's disease (PD) is the second most prevalent neurodegenerative clinical condition after Alzheimer's disease and for the global health system it is essential to identify early signs; however, nowadays it is still a new field of study that needs further development. It has been shown that about $90 \%$ of Parkinson's disease patients also develop deficiencies in her voice, showing symptoms such as monotone speech, low-intensity tone, isolated breaks, inaccurate pronunciation of consonants and prosody problems. Although such problems are identified, only $3 \%$ to $4 \%$ are treated as a voice problem.

In the field of research, time-frequency analysis has proven to be a powerful tool in the processing of acoustic signals, more specifically, the voice processing. With the aim of having clean representations of the spectrum to help with the removal of characteristics and mitigate other resulting problems when using estimated classical methods, it was decided to study the behavior of maximum entropy method, which is currently used in oceanography and astronomy in the study of voice signals.

For these experiments, a database of patients with Parkinson's disease was used. Fifty records of pathological voices and the same amount of healthy voices were studied. Voiced and voiceless segments derived from the word " PA-TA-KA " produced by those patients were analyzed. Energies were estimated from both the Fourier transform and the maximum entropy method. In order to evaluate the performance of the classical methods and the method of maximum entropy, a K-nn classifier was used and success rates were found in around $60 \%$ taking into account the maximum entropy method in both syllables and phonemes.
\end{abstract}

Keywords: Energy, Parkinson's disease, spectrum, K -nn, maximum entropy.

\section{INTRODUCCIÓN}

La Enfermedad de Parkinson (EP) es una de las enfermedades neurodegenerativas más prevalentes en el mundo, con una estadística de ocurrencia que supera los 100/100.000 (1). La EP está caracterizada por la carencia de células dopaminérgicas en el centro del cerebro y sus principales sintomatologías son la presencia de temblor, rigidez y otros desordenes motores. Está demostrado que cerca del $901 \%$ de las personas que padecen la EP también desarrollan un desorden en su voz (2), sin embargo sólo 3\% a 4\% reciben terapia del habla (3). Dado que la edad es el factor más importante para EP y el hecho de que la población mundial está en crecimiento, dichas estadísticas pueden aumentar en un futuro no tan distante (4).

Con el ánimo de realizar un estudio más detallado de las consecuencias de la EP sobre la facultad del habla, se han efectuado diferentes pruebas para extraer los síntomas relacionados a los efectos en los músculos responsables del habla. Por ejemplo en la fonación sostenida (5), el objetivo de la pruebas es pronunciar una oración construida por las unidades lingüísticas pertinentes. Otras pruebas diferentes son útiles para acceder a la disfonía del paciente, sin embargo, su total eficacia para evaluar el avance de la EP no esta todavía determinada. En (6) y (7) proponen una forma alterna para el estudio de la EP, ellos centran su atención a los cambios producidos en las bajas frecuencias del espectro con el fin de caracterizar posibles desplazamientos del velo, lo cual puede ser causado por la falta de control de este miembro. De acuerdo con los resultados, las regiones de baja frecuencia entregan una importante información con la que se pueden caracterizar los desordenes del habla en personas con EP. Por otro lado, la forma en como se obtienen las representaciones en frecuencia es un punto importante para garantizar el buen análisis y la correcta clasificación entre voces sanas y patológicas; la metodología más usada para determinar el espectro de señales acústicas es la implementada a través de la transformada de Fourier (8), de este se derivan métodos no paramétricos como son el periodograma, Blackman-Tukey, entre otros (9). Sin embargo, los espectros obtenidos con estos métodos generalmen- 
te presentan problemas de resolución (10) y suponen una prolongación nula o periódica de la señal a analizar (11) imponiendo limitaciones en los experimentos.

Los métodos paramétricos, como son los casos de Máxima Entropía y máxima verosimilitud proponen espectros con mejor resolución y mitigan las limitaciones impuestas por los métodos basados en la transformada de Fourier; debido a las ventajas mostradas por MEM en la estimación del espectro, los autores (10) y (12) han concentrado su investigación en la búsqueda de futuras aplicaciones para esta técnica. En la actualidad, MEM es utilizado en áreas como la geo-física y astro-física (11) y junto con máxima verosimilitud son los métodos típicamente usados para el análisis de señales geológicas. No obstante, sus ventajas pueden extrapolarse a diferentes campos.

En este artículo se proponen experimentos que permitan evaluar la capacidad discriminante del MEM, con respecto al desempeño que presenta la transformada de Fourier. Para esto se realizan pruebas sobre segmentos sonoros y no sonoros, en los cuales es estimada la energía en frecuencia. Posteriormente, se realiza un análisis estático donde se consideran 4 estadísticos (media, curtosis, desviación estándar y asimetría). El primer experimento considera únicamente los fonemas, mientras que el segundo esta orientado al análisis de silabas; ambos experimentos realizados sobre una base de datos de 50 registros de control y 50 patológicos, teniendo en cuenta la palabra "PA-TA-KA". Por ultimo se usó un clasificador no paramétrico, K-nn, bajo la metodología de validación Bootstrapping, con el fin de mostrar la capacidad discriminante de la transformada de Fourier y del MEM en el proceso de detección de la EP.

El resto del artículo se encuentra organizado de la siguiente manera: en la sección 2 se presenta la metodología que será usada para realizar los experimentos, la sección 3 muestra los detalles del marco experimental resaltando el clasificador y la metodología de validación usada, la sección 4 presenta los resultados obtenidos en los experimentos, finalmente en la sección 5 son presentadas las conclusiones obtenidas de este trabajo.

\section{Metodología}

En esta sección se presenta una descripción de los pasos que se llevaron a cabo en la realización de los experimentos, se dan a conocer también los métodos utilizados para el procesamiento de las señales de voz. La figura 1 resume las etapas que conformaron los experimentos.

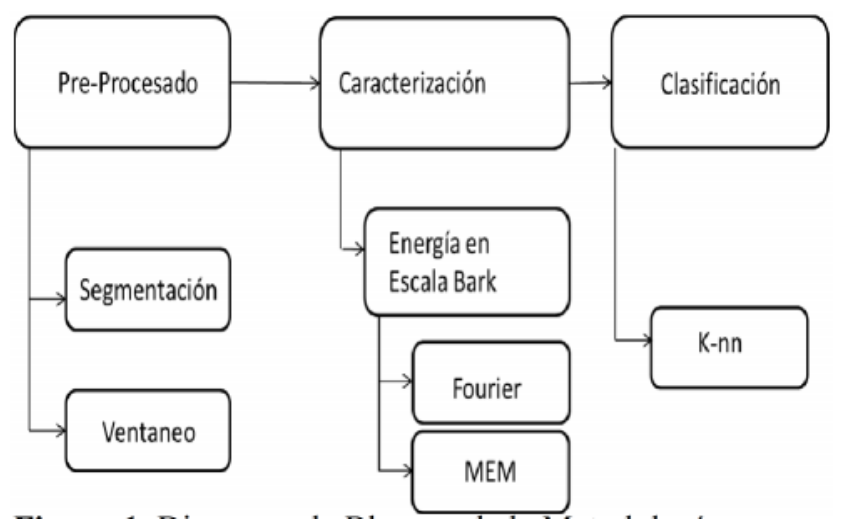

Figura 1. Diagrama de Bloques de la Metodología

A continuación se muestran los aspectos más relevantes de los métodos de estimación espectral usados en los experimentos y la escala de Bark usada para el banco de filtros implementado en el procesamiento de la señal.

\section{A. Estimación de energía en el espectro de Fourier}

La potencia espectral de una señal está dada por la transformada de Fourier de una secuencia de autocorrelación (13), y tomando en consideración que la estimación del espectro es casi directa, el método de Fourier es considerado como el método no-paramétrico más utilizado en el análisis de señales biomédicas. Sea $x(n)$ el registro de voz a analizar, su transformada de Fourier esta dada por la ecuación [1]:

$$
Y(f)=\prod_{J=1}^{\mathrm{N}} x(j) w_{n}^{(j-1)(k-1)}
$$

donde $N$ es el número de puntos de la transformada.

$Y(f)$ entonces será la representación en frecuencia del registro de voz. Por tanto la energía, tomando en cuenta una separación $\mathrm{T}_{\mathrm{s}}$, estará dada por la ecuación [2]:

$$
E_{F F T}=\frac{1}{N f_{s}} I_{n=0}^{N}|Y(f)|^{2}
$$

Sin embargo, existen algunas limitaciones para esta estimación, que pueden acarrear problemas en el análisis. Tales como: 
El número de datos tomados para la estimación del espectro con Fourier no puede ser infinita debido a que se necesitaría una máquina con memoria infinita para realizarla, por tal motivo los puntos para el análisis se toman por espacios de tiempo en los cuales puede darse el caso de que los datos tomados no sean coherentes con el proceso que se quiera analizar, como es el caso de procesos de corta duración (13).

El ruido inherente de la señal puede tener influencia en la estimación (13).

Suponer una prolongación nula o periódica de la señal a analizar (11).

\section{B. Estimación de energía en espectro de Máxima Entropía}

Con el fin de suplir, o al menos mitigar, las limitaciones de los métodos no paramétricos para la estimación del espectro, como lo es el caso de Fourier, métodos paramétricos como el de Máxima Entropía proponen alternativas que resultan útiles para la ejecución de experimentos (9). Una de las principales ventajas que aporta MEM es mejorar la resolución del espectro en relación a la transformada de Fourier, tal como se puede notar en la figura $2(10)$.
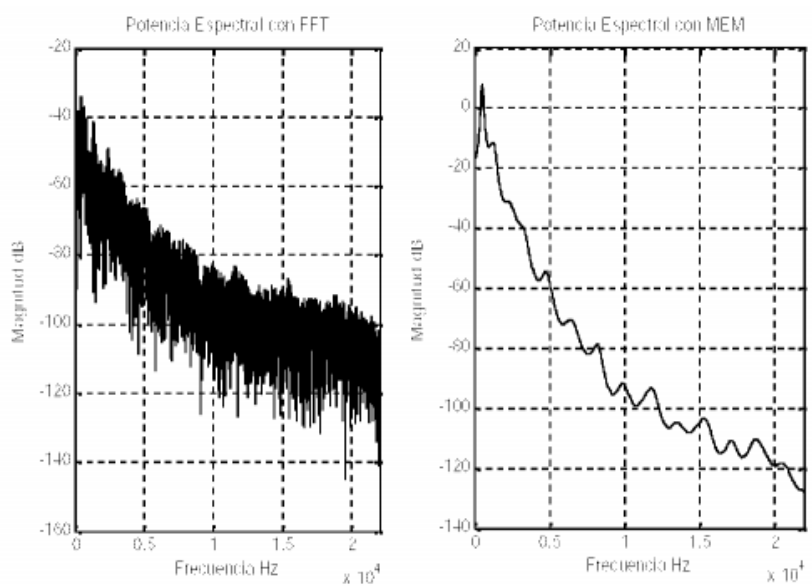

Figura 2. Contraste de resolución entre MEM y transformada de Fourier

En cuanto al manejo de señales como la de voz, al tomar un tubo acústico como modelo para el tracto vocal, se impone un modelo auto-regresivo (AR) para la forma de onda de la voz (13).Por lo tanto, en un intervalo de tiempo en el que la señal de voz sea considerada estacionaria, como es el caso de las ventanas temporales resultantes del análisis en tiempo corto, el espectro será de la forma expresada en la ecuación [3] (13):
$P_{x}\left(e^{j \omega}\right)=\frac{|b(0)|^{2}}{|1+|,\left.\sum_{k=1}^{p} a_{p}(k) e^{-j k w}\right|^{2}}$

Aplicando un método paramétrico es posible incorporar un modelo AR para el cálculo del espectro mejorando así, la resolución. Luego de elegir el modelo, se debe obtener el orden apropiado que puede ser elegido con un previo conocimiento de la señal que se quiere procesar, o basándose en resultados de experimentos hechos con diferentes órdenes. Posteriormente, se procede a calcular sus parámetros según los datos del proceso (13).

En algunos casos, con $N$ datos y $k$ retrasos, la representación sólo puede ser obtenida para ||$k \mid<N$, por lo tanto, la AR para $|k| \geq N$ es cero. En contraste, MEM realiza una extrapolación más acertada que evita dichos valores llevados a cero y por tanto es posible determinar un espectro más preciso. La extrapolación se basa en un concepto extraído de la teoría dela información, considerando la entropía como una medida de aleatoriedad, y bajo este concepto Burg planteo el siguiente análisis (14).

La extrapolación por máxima entropía es equivalente a encontrar la secuencia de $A R r_{e}^{\wedge}(k)$ que haga la señal $x(n)$ lo más aleatoria posible.

1. Se encontró un modelo todos polos, usando el método de auto-correlación $x(n)$.

2. La entropía está dada por la expresión [4] (13):

$$
H(x)=\frac{1}{2 \pi} \#_{-r} \ln P_{x}\left(e^{j \omega}\right) d \omega
$$

Donde se maximiza, con el fin de extrapolar la autocorrelación para los valores de $|k| \geq N$, y luego de un tratamiento matemático es obtiene el espectro en potencia expresada como se muestra en la ecuación [5] (13).

$$
\widehat{P}_{m e m}\left(e^{j \omega}\right)=\frac{\dagger_{N}}{\left|\mathbf{f}^{H} a^{N}\right|^{2}}
$$

En donde:

$$
\epsilon_{N}=r_{x}(0)+\prod_{k=1}^{N} a_{N}(k) r_{x}^{*}(k)=|b(0)|^{2}
$$

y $a_{N}$ es la solución para el sistema [6] (13): 


$\left[\begin{array}{cccc}r_{x}(0) & r_{x}^{*}(1) & \ldots & r_{x}^{*}(1) \\ r_{x}(1) & r_{x}^{*}(0) & \ldots & r_{x}^{*}(N-1) \\ \vdots & \vdots & \vdots & \vdots \\ r_{x}(N) & r_{x}^{*}(N-1) & \ldots & r_{x}^{*}(0)\end{array}\right]\left[\begin{array}{c}1 \\ a_{N}(1) \\ \ldots \\ a_{N}(N)\end{array}\right]=\varepsilon_{P}\left[\begin{array}{c}1 \\ 0 \\ \ldots \\ 0\end{array}\right]$

Por último, todos los parámetros $\mathbf{a}_{N}$ fueron calculados resolviendo las ecuaciones de Yule-Walker.

La figura 3 resume el método usado para el cálculo de la potencia espectral en el MEM.

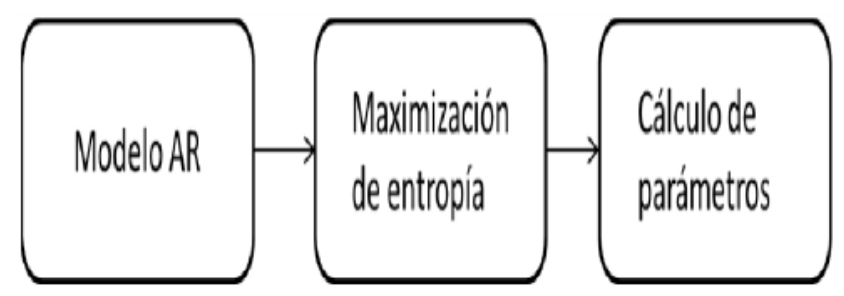

Figura 3. Diagrama de bloques MEM

\section{Escala de Bark}

Se define a partir de las mediciones de las frecuencias del sistema auditivo humano, encontradas por el ancho de las bandas criticas, (1 Banda crítica=1 Bark), la escala varía entre 1 a 24 Bark (15).

Las bandas críticas se refieren a los rangos de frecuencia correspondientes a las regiones de la membrana basilar que son estimuladas a frecuencias específicas (16). Los límites críticos, no se asignan según la frecuencia sino que dependen de estímulos particulares; por tal razón existen cuatro estrategias para la localización de dichos límites, estas estrategias son: el umbral, el enmascaramiento, la fase y la sonoridad.

La escala de Bark presenta un comportamiento lineal por debajo de los $500 \mathrm{~Hz}$ y logarítmico a medida que aumenta la frecuencia, además está relacionada con las distancias entre las frecuencias en la membrana basilar del oído (15).

Existe una relación entre la frecuencia de Bark y la frecuencia lineal, dicha relación está dada por le expresión [7]:

$\operatorname{Bark}(f)=13 \tan ^{-1}(0.0076 f)+3,5 \tan ^{-1}\left(\frac{\mathrm{f}}{75000}\right)^{2}$

Donde $f$ es la frecuencia en la escala lineal y $\operatorname{Bark}(f)$ es la frecuencia resultante en la escala de Bark.

\section{Marco Experimental A. Base de Datos}

Para este experimento fue usada una base de datos de pacientes que presentan la EP, con 50 registros de voces patológicas y 50 registros de voces sanas. Todos los registros se grabaron bajo las mismas condiciones acústicas a una frecuencia de muestreo de $44.1 \mathrm{KHz}$ con una resolución de 16 bits. La base de datos fue balanceada por género y edad: las edades de los pacientes hombres están en un rango de 33 a 77 años (con una media de $62.2 \pm 11.2$ ) y las edades de las pacientes mujeres en un rango de 44 a 75 años (con una media de $60.1 \pm 7.8$ ). Para el caso de los hombres sanos, están en un rango de 31 a 86 años (con una media de $61.2 \pm 11.3$ ) y para mujeres sanas en un rango de 43 a 76 años (con una media de $60.73 \pm 7.7$ ). Todos los pacientes fueron diagnosticados por neurólogos expertos; el valor medio de las evaluaciones de a cuerdo a las escalas UPDRS-III y Hoehn \& Yhar son 38.2 y 2.3 , respectivamente. Ninguna de las personas en el grupo de control tiene historial de sintomatología relacionada con la EP o algún tipo de desorden motriz (Mayores detalles en (16)). Los registros consisten en la repetición de la palabra "PA-TA-KA". La base de datos fue construida por la Universidad de Antioquia en Medellín Colombia.

\section{B. Procesado}

A cada registro de voz extraído de la base de datos se le realizó una segmentación haciendo uso del programa Praat y el pluging Easyaling (18); dicho proceso es realizado en tres pasos: Macro-segmentación, conversión ortográfica a fonética y segmentación de fonemas.

- Macro-Segmentación: En este paso, se indica en el audio de voz en que intervalo se producen las frases dentro de un oración.

- Conversión ortográfica a fonética: Este paso se realiza la conversión ortográfica a transcripción fonética de acuerdo al alfabeto fonético SAMPA Speech Assessment Methods Phonetic Alphabet.

- Segmentación de fonemas: Por último, en el archivo de audio son definidos los intervalos a nivel de fonemas, silabas y palabras. Un sistema de reconocimiento es utilizado para estimar la duración temporal de la palabra, silaba o fonema que hace parte de cada frase.

La segmentación se efectuó en segmentos sonoros y 
no sonoros, separados en silabas y fonemas. Con el fin de hacer una análisis a tiempo corto, los segmentos fueron divididos en ventanas de 40ms de duración con solape del 50\%; sobre cada ventana se estima la energía a partir de dos técnicas, la transformada de Fourier y el MEM. Adicionalmente, se aplica un banco de 11 filtros en la escala de Bark, con el fin de obtener la contribución de la energía en diferentes bandas de frecuencia.

\section{Experimentos}

Para este trabajo se tiene en cuenta 2 experimentos principales. El primero, busca evaluar la capacidad discriminante de los fonemas en el proceso de detección de la EP. El segundo, mide la capacidad discriminante al evaluar únicamente las silabas. Ambos experimentos se basan en la estimación de la energía a partir de la transformada de Fourier y el MEM, teniendo en cuenta la contribución que ofrecen bandas especificas de frecuencia a partir de un banco de 11 filtros en la escala de Bark.

Debido a que para cada segmento aparecen tantos valores como ventanas, para cada característica deben ser calculados estadísticos, tales como: Media, desviación estándar, curtosis y asimetría.

\section{Clasificación}

Para el análisis de los resultados arrojados por los experimentos se usó un clasificador no paramétrico, tal como el K vecinos más cercanos (K-nn), el cual se trabajó bajo la metodología de validación bootstrapping; se tomó un 70\% de los registros para el entrenamiento del clasificador y el $30 \%$ restante para pruebas. El procedimiento se reprodujo diez veces, y para cada repetición el conjunto de datos para entrenamiento y validación fue elegido aleatoriamente, esto con el ánimo de obtener intervalos de confianza para la estimación del desempeño general de los experimentos propuestos.

\section{Resultados}

La Tabla 1 muestra los resultados obtenidos al evaluar la capacidad discriminante de los fonemas presentes en la palabra "PA-TA-KA", teniendo en cuenta dos métodos para la estimación del espectro y la posterior estimación de la energía. En ambos métodos se aplica un banco de filtros en la escala de Bark, permitiendo evaluar la contribución que tienen 11 bandas de frecuencia. La intensión de este experimento es determinar que fonema presenta mayor contribución al proceso de clasificación entre una voz sana y una patológica.

Tabla 1. Desempeño del experimento 1, considerando fonemas

\begin{tabular}{ccccc}
\hline Fonema & Método & Acierto & Especificidad & Sensibilidad \\
\hline$/ \mathbf{p} /$ & Fourier & $0.5257 \pm 0.0521$ & $0.5262 \pm 0.0452$ & $0.5270 \pm 0.0747$ \\
& MEM & $0.5114 \pm 0.0546$ & $0.5229 \pm 0.0759$ & $0.5062 \pm 0.0585$ \\
$/ \mathbf{a} /$ & Fourier & $0.5414 \pm 0.0608$ & $0.5519 \pm 0.0504$ & $0.5561 \pm 0.1193$ \\
& MEM & $0.5471 \pm 0.0556$ & $0.5522 \pm 0.0502$ & $0.5504 \pm 0.0748$ \\
$/ \mathbf{t} /$ & Fourier & $0.4929 \pm 0.0588$ & $0.4959 \pm 0.0565$ & $0.4960 \pm 0.0711$ \\
& MEM & $0.5329 \pm 0.0357$ & $0.5356 \pm 0.0356$ & $0.5461 \pm 0.0726$ \\
$/ \mathbf{a} /$ & Fourier & $0.5171 \pm 0.0699$ & $0.5324 \pm 0.0945$ & $0.5059 \pm 0.0547$ \\
& MEM & $\mathbf{0 . 5 6 2 9} \pm \mathbf{0 . 0 4 3 7}$ & $\mathbf{0 . 5 4 8 4} \pm \mathbf{0 . 0 4 0 4}$ & $\mathbf{0 . 5 8 9 8} \pm \mathbf{0 . 0 8 0 8}$ \\
$/ \mathbf{k} /$ & Fourier & $0.5486 \pm 0.0531$ & $0.5635 \pm 0.0789$ & $0.5491 \pm 0.0585$ \\
& MEM & $0.5529 \pm 0.0650$ & $0.5485 \pm 0.0720$ & $0.5606 \pm 0.0726$ \\
$/ \mathbf{a} /$ & Fourier & $\mathbf{0 . 5 6 0 0} \pm \mathbf{0 . 0 6 4 2}$ & $\mathbf{0 . 5 6 4 9} \pm \mathbf{0 . 0 8 9 7}$ & $\mathbf{0 . 5 6 9 1} \pm \mathbf{0 . 0 6 2 3}$ \\
& MEM & $0.5043 \pm 0.0539$ & $0.5150 \pm 0.0545$ & $0.5051 \pm 0.0830$ \\
\hline
\end{tabular}

Puede notarse que el mejor resultado para el MEM se encuentra al evaluar la segunda vocal/a/, mientras que para el método basado en la transformada de Fourier su mejor resultado se encuentra en la tercer vocal /a/.
Por otra parte, para el segundo experimento la Tabla 2 muestra los resultados obtenidos al evaluar únicamente las características provenientes de las sílabas presentes en la palabra "PA-TA-KA". 
Evaluación de métodos de fourier para la detección automática de la enfermedad de Parkinson. Arias et al.

Tabla 2. Desempeño del experimento 2, considerando sílabas

\begin{tabular}{|c|c|c|c|c|}
\hline Sílaba & Método & Acierto & Especificidad & Sensibilidad \\
\hline \multirow[t]{2}{*}{$/ \mathbf{p a} /$} & Fourier & $0.5371 \pm 0.0518$ & $0.5338 \pm 0.0570$ & $0.5433 \pm 0.0666$ \\
\hline & MEM & $0.5471 \pm 0.0547$ & $0.5468 \pm 0.0578$ & $0.5742 \pm 0.1042$ \\
\hline \multirow[t]{2}{*}{$/ \mathbf{t a} /$} & Fourier & $0.530 \pm 0.0585$ & $0.5295 \pm 0.0640$ & $0.5554 \pm 0.0893$ \\
\hline & MEM & $0.5586 \pm 0.0545$ & $0.5623 \quad 0.0510$ & $0.5575 \pm 0.0922$ \\
\hline \multirow[t]{2}{*}{$/ \mathbf{k a} /$} & Fourier & $0.5971 \pm 0.0586$ & $0.6302 \pm 0.1174$ & $0.5850 \pm 0.0419$ \\
\hline & MEM & $0.5814 \pm 0.0363$ & $0.5801 \pm 0.0710$ & $0.6114 \pm 0.0632$ \\
\hline
\end{tabular}

Al considerar únicamente las sílabas, se pudo notar que tanto para MEM como para el método basado en la transformada de Fourier se presenta el mejor desempeño con la sílaba $/ \mathrm{ka} /$, con tasas de acierto superiores al $58 \%$.

Tabla 3. Desempeño al unir los espacios de representación

\begin{tabular}{ccccc}
\hline Unión & Método & Acierto & Especificidad & Sensibilidad \\
\hline Fonemas & Fourier & $0.5371 \pm 0.0468$ & $0.5510 \pm 0.0714$ & $0.5334 \pm 0.0456$ \\
& MEM & $\mathbf{0 . 5 7 1 4} \pm \mathbf{0 . 0 2 9 4}$ & $\mathbf{0 . 6 0 7 7} \pm \mathbf{0 . 0 6 9 2}$ & $\mathbf{0 . 5 6 2 6} \pm \mathbf{0 . 0 4 2 3}$ \\
Sílabas & Fourier & $0.5271 \pm 0.0742$ & $0.5287 \pm 0.0727$ & $0.5285 \pm 0.0860$ \\
& MEM & $\mathbf{0 . 5 8 7 1} \pm \mathbf{0 . 0 4 8 8}$ & $\mathbf{0 . 6 2 4 5} \pm \mathbf{0 . 0 6 2 3}$ & $\mathbf{0 . 5 6 8 8} \pm \mathbf{0 . 0 4 9 3}$ \\
\hline
\end{tabular}

\section{Conclusiones}

En este trabajo se ha presentado una metodología para la detección automática de la enfermedad de Parkinson, por medio del análisis de señales de voz, estimando características de energía a partir del estudio clásico de la transformada de Fourier y por medio de un método novedoso basado en la máxima entropía, evaluando su capacidad discriminante.

En el primer experimento se pudo notar que los fonemas sonoros presentan un mejor desempeño, en comparación con los segmentos no sonoros. Los aciertos más altos fueron alcanzados con el fonema $/ \mathrm{a} /$, con tasas de hasta $56 \%$. En cuanto al experimento que considera las sílabas, se presentan los mejores resultados en /ka/, tanto para Fourier como para MEM, alcanzando una tasa de acierto de hasta $59 \%$ en el mejor de los casos. Por último, al considerar la unión de los espacios de representación se pudo notar que MEM presenta los mejores desempeños tanto en fonemas como en sílabas, alcanzando una tasa de $58 \%$ para las sílabas.

Aunque la metodología no presenta resultados cerca-
Finalmente, con el ánimo de evaluar espacios de representación que consideren la unión entre los 5 fonemas, también entre las 3 sílabas, la Tabla 3 muestra los resultados al unir dichos espacios de representación. nos al estado del arte, se podría considerar otro tipo de características que tengan en cuenta la naturaleza de los segmentos, ya que existen características propias para segmentos sonoros y otras para segmentos no sonoros.

Como trabajo futuro se buscará la implementación de características presentadas en el estado del arte, que hayan presentado mejores resultados en términos de acierto, considerando la naturaleza del segmento y basados en el método de máxima entropía.

\section{Agradecimientos}

Este trabajo está financiado por COLCIENCIAS a través del proyecto número 111556933858. Adicionalmente, Elkyn Alexander Belalcazar Bolaños está beneficiado por el programa de Jóvenes Investigadores e Innovadores COLCIENCIAS 2014. Juan Rafael Orozco está favorecido por la convocatoria 528 para estudios de doctorado en Colombia 2011, financiado por COLCIENCIAS.

Los autores agradecen al CODI, estrategia de sostenibilidad 2014-2015 de la Universidad de Antioquia, por el apoyo para el desarrollo de este trabajo. 


\section{BIBLIOGRAFÍA}

1. A. S. von Campenhausen, B.Bornschein, R.Wick, K.Botzel, C. Sampaio, W. Poewe, W. Oertel, U. Siebert, K. Berger, R. Dodel. Prevalence and Incidence of Parkinson's Disease in Europe. Eur. Neuropsychophar- macol 2005; 15: 473-490.

2. A. Ho, R. Iansek, C. Marigliani, J. Bradshaw, S. Gates. Speech Impairment in a Large Sample of Patients With Parkinson's Disease. Behavioral Neurology 1998; 11: 131-137.

3. L. Ramig, C. Fox, S. Shimon. Speech Treatment for Parkinson's Disease. Expert Review Neurotherapeutics 2008; 8(2): 297-309.

4. S. D. Eeden, C. Tanner, A. L. Bernstein, R. Fross, A. Leim-peter, D. A. Bloch, L. Nelson. Incidence of parkinsonâ $€^{\mathrm{TM}_{\mathrm{S}}}$ Disease: Variation by Age, Gender, and Race/Ethnicity. Am. J. Epidem 2003; 157 : 1015-1022.

5. P. Dejonckere, P. Bradley, P. Clemente, G. Cornut, L. Crevier-Buchman, G. Friedrich, P. V. D. Heyning, M. Remacle, V. Woisard. A basic protocol for functional assessment of voice pathology, especially for investigating the efficacy of (phonosurgical) treatments and evaluating new assessment techniques. Guideline elaborated by the Committee on Phoniatrics of the European Laryngological Society (ELS), Eur Arch Otorhinolaryngol 2001; 258(7): 77-82.

6. P. Vijayalakshmi, M. Reddy. Assessment of dysarthric speech and an analysis on velopharyngeal incompetence', Proceedings of the IEEE Engineering in Medicine and Biology Society (EMBS). 3 September $2006: 3759-3762$.

7. E. Belalcazar-Bolaños, J. Orozco-Arroyave, J. Vargas-Bonilla, J. Arias- Londoño, C. CastellanosDomí $\neg$ nguez, E. Nöth. Low-frequency of speech for automatic detection of parkinson's disease. Lecture Notes in Computer Science, 2013; 7930: 283-292.

8. L. R. Rabiner, R. W. Schafer. Introduction to digital speech processing. Foundations and Trends in Signal Processing 2007; 1(1): 1-194.

9. L. Baghai-Ravary, S. Beet. Multistep coding of speech parameters for compression. IEEE Trans. Speech Audio Process 1998; 6(5): 435-444.

10. J. Lim, N. Malik. Maximum entropy power spectrum estimation of signals with missing correlation points. IEEE Trans. Acoust., Speech, Signal Process 1981; 29(6): 1215-1217.

11. J. J. Egozcue. Una interpretación del análisis espectral de máxima entropía. Revista de Obras Públicas 1980; 127: 167-172.

12. B. Choi. An information-theoretic derivation of the 2- D maximum entropy spectrum. IEEE Signal Process. Lett 1998; 5(10): 271-272.

13. M. H. Hayes. Statistical digital signal processing and modeling. New York: 1996.

14. G. Carayannis, D. Manolakis, N. Kalouptsidis. A fast sequential algorithm for least-squares filtering and prediction. IEEE Trans. Acoust. Speech, Signal Process 1983; 31(6): 1394-1402.

15. T. Villa-Canas, E. Belalcazar-Bolaños, S. Bedoya-Jaramillo, J. Garces, J. Orozco-Arroyave, J. AriasLondono, J. Vargas-Bonilla. Automatic detection of laryngeal pathologies using cepstral analysis in mel and bark scales', XVII Symposium of Image, Signal Processing, and Artificial Vision (STSIVA). 14 September 2012: 116-121.

16. W. Brent. Perceptually based pitch scales in cepstral techniques for percussive timbre identification. University of Michigan Library: 2009.

17. J. R. Orozco-Arroyave, J. D. Arias-Londoño, J. F. Vargas-Bonilla, M. C. Gonzalez-Rativa, E. Nöth. New spanish speech corpus database for the analysis of people suffering from parkinson's disease', Proceedings of the 9th Language Resources and Evaluation Conference, LREC 2014. 31 May 2014.

18. J.-P. Goldman. Easyalign: an automatic phonetic alignment tool under praat proceedings of interspeech', September 2011, Firenze, Italy. 